Article

\title{
Geodesic Chord Property and Hypersurfaces of Space Forms
}

\author{
Dong-Soo Kim ${ }^{1}$, Young Ho Kim ${ }^{2, *}$ and Dae Won Yoon ${ }^{3}$ (i) \\ 1 Department of Mathematics, Chonnam National University, Gwangju 61186, Korea \\ Department of Mathematics, Kyungpook National University, Daegu 41566, Korea \\ 3 Department of Mathematics Education and RINS, Gyeongsang National University, Jinju 52828, Korea \\ * Correspondence: yhkim@knu.ac.kr; Tel.: +82-53-950-7325
}

Received: 22 July 2019; Accepted: 14 August 2019; Published: 16 August 2019

\begin{abstract}
In the Euclidean space $\mathbb{E}^{n}$, hyperplanes, hyperspheres and hypercylinders are the only isoparametric hypersurfaces. These hypersurfaces are also the only ones with chord property, that is, the chord connecting two points on them meets the hypersurfaces at the same angle at the two points. In this paper, we investigate hypersurfaces in nonflat space forms with the so-called geodesic chord property and classify such hypersurfaces completely.
\end{abstract}

Keywords: geodesic chord property; hypersphere; hyperbolic space; isoparametric hypersurface

\section{Introduction}

A circle in the plane $\mathbb{E}^{2}$ is characterized as a closed curve with the chord property that the chord connecting any two points on it meets the curve at the same angle at the two end points ([1], pp. 160-162).

For space curves, B.-Y. Chen et al. showed that a $W$-curve is characterized as a curve in the Euclidean space $\mathbb{E}^{n}$ with the property that the chord joining any two points on the curve meets the curve at the same angle at the two points, that is, as a curve in the Euclidean space $\mathbb{E}^{n}$ with the chord property ([2]).

For hypersurfaces in the Euclidean $n$-space $\mathbb{E}^{n}$ which satisfies the chord property, D.-S. Kim and Y. H. Kim established the following classification theorem ([3]). See also [4-6].

Proposition 1. Let us consider a hypersurface $M$ in the Euclidean space $\mathbb{E}^{n}$. Then the following are equivalent:

1. M satisfies the chord property.

2. The Gauss map $G$ of $M$ satisfies $G(x)=A x+b$ for some $n \times n$ matrix $A$ and a vector $b \in \mathbb{E}^{n}$.

3. $M$ is an isoparametric hypersurface.

4. $M$ is contained in one of the following hypersurfaces: $\mathbb{E}^{n-1}, S^{n-1}(r), S^{p-1}(r) \times \mathbb{E}^{n-p}$.

In this paper, we consider hypersurfaces in the $n$-dimensional space form $\bar{M}^{n}(c)$ with nonzero constant sectional curvature $c$. The hypersurface $M$ is said to satisfy geodesic chord property if the geodesic chord in the ambient space $\bar{M}^{n}(c)$ joining any two points on $M$ meets the hypersurface at the same angle at the two points. Note that a geodesic chord in the ambient space $\bar{M}^{n}(c)$ is defined by a segment of a geodesic of $\bar{M}^{n}(c)$ with two end points. When $c>0$, that is, the ambient space $\bar{M}^{n}(c)$ is a hypersphere $S^{n}$ in the Euclidean $(n+1)$-space $\mathbb{E}^{n+1}$, we consider only two points which are not antipodal to each other.

First of all, in Section 2, we study spherical hypersurfaces in the $n$-dimensional unit sphere $S^{n}$ which satisfy the geodesic chord property and then classify such hypersurfaces. Next, in Section 3, 
we study and classify completely the hypersurfaces in the $n$-dimensional hyperbolic space $H^{n}$ with the geodesic chord property, which is imbedded in the Minkowski space $\mathbb{E}_{1}^{n+1}$.

In the Euclidean space $\mathbb{E}^{n+1}$, some characterizations of hyperspheres, ellipsoids, elliptic paraboloids and elliptic hyperboloids were given in [7-10], respectively. In the Minkowski space $\mathbb{E}_{1}^{n+1}$, a few characterizations of hyperbolic spaces were also established in [11].

Throughout this article, we assume that all objects are smooth and connected unless otherwise mentioned.

\section{Spherical Hypersurfaces}

In this section, we consider a hypersurface $M$ in the unit hypersphere $S^{n} \subset \mathbb{E}^{n+1}$ centered at the origin.

For each point $x \in M$, we denote by $G(x)$ the unit normal to $T_{x} M$ in the unit hypersphere $S^{n}$. Then $G: M \rightarrow S^{n}$ is called the Gauss map of $M$ in the unit hypersphere $S^{n}$. For an orthonormal local frame field $\left\{e_{1}(x), \ldots, e_{n-1}(x)\right\}$ on $M,\left\{e_{1}(x), \ldots, e_{n-1}(x), G(x), x\right\}$ forms an orthonormal basis of $T_{x} \mathbb{E}^{n+1}$. We denote by $A(x)$ the $(n+1) \times(n-1)$ frame matrix $\left[e_{1}(x), \ldots, e_{n-1}(x)\right]$ with column vectors $e_{1}(x), \ldots, e_{n-1}(x)$.

For any two points $x, y \in M \subset S^{n}$ with $x+y \neq 0$, we denote by $\theta \in(0, \pi)$ the angle between $x$ and $y$ in the ambient space $\mathbb{E}^{n+1}$. Then the unit speed geodesic chord $\gamma(t)$ with $\gamma(0)=x$ and $\gamma(\theta)=y$ is given by $\gamma(t)=\cos t x+\sin t v$, where we put

$$
v=v_{x, y}=-\cot \theta x+\csc \theta y .
$$

The geodesic chord has initial velocity $v=v_{x, y}$ at $x$.

The projection $P_{x}(v)$ of $v$ onto the tangent space $T_{x} M$ is given by

$$
P_{x}(v)=A(x) \alpha,
$$

where $\alpha$ is a vector in $\mathbb{E}^{n-1}$. In order to determine $\alpha \in \mathbb{E}^{n-1}$, first note that $v-P_{x}(v)$ is perpendicular to $T_{x} M$. Then, (2) shows that

$$
A(x)^{t}(v-A(x) \alpha)=0,
$$

where $A(x)^{t}$ denotes the transpose of $A(x)$. Since $A(x)^{t} A(x)$ is the $(n-1) \times(n-1)$ identity matrix $I_{n-1}$, we get from (3)

$$
\alpha=A(x)^{t} v .
$$

Thus, the projection $P_{x}(v)$ of $v$ into the tangent space $T_{x} M$ is given by

$$
P_{x}(v)=P(x) v,
$$

where $P(x)$ denotes the $(n+1) \times(n+1)$ matrix given by

$$
P(x)=A(x) A(x)^{t} .
$$

Now, for later use we prove a lemma as follows.

Lemma 1. For a spherical hypersurface $M$, the following are equivalent:

1. M satisfies the geodesic chord property.

2. For points $x, y \in M$, we have

$$
\left|A(x)^{t} y\right|=\left|A(y)^{t} x\right| .
$$

3. For points $x, y \in M$, we have

$$
\langle G(x), y\rangle=\epsilon\langle x, G(y)\rangle,
$$


where $G(x)$ denotes the Gauss map of $M$ and $\epsilon= \pm 1$.

Proof. For two points $x, y \in M \subset S^{n}$ with $x+y \neq 0$, we denote by $\theta$ the angle between $x$ and $y$ as above. If we let $\phi$ denote the angle between the geodesic chord $\gamma$ from $x$ to $y$ and the tangent plane $T_{x} M$ at the point $x$, then together with (1), (5) and (6) show that

$$
\cos \phi=|P(x) v|=\csc \theta|P(x) y|,
$$

where the second equality follows from $A(x)^{t} x=0$, because $x$ is orthogonal to the tangent plane $T_{x} M$. Using $A(x)^{t} A(x)=I_{n-1}$, we see that

$$
|P(x) y|=\left|A(x)^{t} y\right|
$$

Hence we have

$$
\cos \phi=\csc \theta\left|A(x)^{t} y\right| .
$$

Similarly, by interchanging $x$ and $y$ in the above discussions the angle $\psi$ between the geodesic chord from $y$ to $x$ and the tangent plane $T_{y} M$ at the point $y$ is given by

$$
\cos \psi=\left|P(y) v_{y, x}\right|=\csc \theta\left|A(y)^{t} x\right|,
$$

where we use

$$
v_{y, x}=-y \cot \theta+x \csc \theta .
$$

Together with (10)-(12) imply that (1) and (2) in Lemma 1 are equivalent to each other for $x, y \in M$ with $x+y \neq 0$. By continuity, (8) holds for all $x, y \in M$.

If we consider the following expression of $y$

$$
y=\sum_{i=1}^{n-1} y_{i} e_{i}(x)+y_{n} G(x)+y_{n+1} x
$$

then we have

$$
y_{i}=\left\langle y, e_{i}(x)\right\rangle, \quad i=1,2, \ldots, n-1
$$

and

$$
y_{n}=\langle y, G(x)\rangle, \quad y_{n+1}=\langle y, x\rangle .
$$

Hence we get from (14)-(16)

$$
\left|A(x)^{t} y\right|^{2}=\sum_{i=1}^{n-1} y_{i}^{2}=|y|^{2}-\langle y, G(x)\rangle^{2}-\langle y, x\rangle^{2},
$$

where the first equality follows from $A(x)^{t} y=\left(y_{1}, \ldots, y_{n-1}\right)^{t}$.

By interchanging $x$ and $y$, we obtain from (17)

$$
\left|A(y)^{t} x\right|^{2}=|x|^{2}-\langle x, G(y)\rangle^{2}-\langle x, y\rangle^{2} .
$$

Combining (17) and (18) shows that (2) and (3) are equivalent to each other. This completes the proof of Lemma 1.

Remark 1. Without using (7), we may prove the equivalence of (1) and (3) in Lemma 1. Since (7) holds for spherical submanifolds with geodesic chord property, it is useful in the study of such spherical submanifolds. 
We now suppose that a hypersurface $M$ in the unit hypersphere $S^{n} \subset \mathbb{E}^{n+1}$ satisfies the geodesic chord property. We may assume, without loss of generality, that $M$ lies fully in the Euclidean space $\mathbb{E}^{n+1}$, which means that $M$ is not contained in any hyperplane of $\mathbb{E}^{n+1}$. Otherwise, it is an open part of a small sphere $S^{n-1}(r) \subset S^{n}$ for some $r \in(0,1]$. Hence, on $M$ there exist points $y_{0}, y_{1}, \ldots, y_{n+1}$ such that the set $\left\{A_{j} \mid A_{j}=y_{j}-y_{0}, j=1,2, \ldots, n+1\right\}$ spans the Euclidean space $\mathbb{E}^{n+1}$.

It follows from (3) of Lemma 1 that we have

$$
\left\langle G(x), y_{0}\right\rangle=\epsilon\left\langle G\left(y_{0}\right), x\right\rangle
$$

and

$$
\left\langle G(x), y_{j}\right\rangle=\epsilon\left\langle G\left(y_{j}\right), x\right\rangle, j=1,2, \ldots, n+1,
$$

where $\epsilon= \pm 1$. Hence we obtain from (19) and (20)

$$
\left\langle G(x), A_{j}\right\rangle=\left\langle B_{j}, x\right\rangle, j=1,2, \ldots, n+1,
$$

where we put for $j=1,2, \ldots, n+1$

$$
A_{j}=y_{j}-y_{0}, B_{j}=\epsilon G\left(y_{j}\right)-\epsilon G\left(y_{0}\right) .
$$

We denote by $A$ the $(n+1) \times(n+1)$ matrix defined by

$$
A^{t}=\left[B_{1}, B_{2}, \ldots, B_{n+1}\right]\left[A_{1}, A_{2}, \ldots, A_{n+1}\right]^{-1},
$$

where $\left[A_{1}, A_{2}, \ldots, A_{n+1}\right]$ is the $(n+1) \times(n+1)$ matrix consisting of columns $A_{1}, A_{2}, \ldots, A_{n+1}$, etc. Then we have from (21)

$$
G(x)=A x .
$$

Therefore, we get the following lemma.

Lemma 2. Suppose that a hypersurface $M$ in the unit hypersphere $S^{n} \subset \mathbb{E}^{n+1}$ satisfies the geodesic chord property. If the hypersurface $M$ lies fully in $\mathbb{E}^{n+1}$, then for an $(n+1) \times(n+1)$ matrix $A$, the Gauss map $G(x)$ satisfies $G(x)=A x$.

Remark 2. If the hypersurface $M$ does not lie fully in $\mathbb{E}^{n+1}$, that is, $M$ is contained in a hyperplane, then it is an open part of a small sphere $S^{n-1}(r) \subset S^{n}$ for some $r \in(0,1]$. In fact, for some unit vector $a \in \mathbb{E}^{n+1}$ we have

$$
M \subset\left\{x \in S^{n} \mid\langle x, a\rangle=\cos \theta\right\},
$$

where $\cos \theta=\sqrt{1-r^{2}}$. Then, we get

$$
G(x)=-\cot \theta x+\csc \theta a .
$$

Thus, $M$ satisfies $G(x)=A x+b$ for $A=-\cot \theta I$ and $b=\csc \theta a$.

Finally, we need the following proposition, which was proved in [12].

Proposition 2. A hypersurface $M$ in the unit hypersphere $S^{n} \subset \mathbb{E}^{n+1}$ satisfies for an $(n+1) \times(n+1)$ matrix $A$ and $a$ vector $b \in \mathbb{E}^{n+1}$

$$
G(x)=A x+b
$$

if and only if it is an open part of either a sphere $S^{n-1}(r)$ or a product $S^{p}\left(r_{1}\right) \times S^{n-p-1}\left(r_{2}\right)$ of spheres with $r_{1}^{2}+r_{2}^{2}=1$. 
It follows from Proposition 2 that the hypersurface $M$ is an open part of either a sphere $S^{n-1}(r)$ or a product $S^{p}\left(r_{1}\right) \times S^{n-p-1}\left(r_{2}\right)$ of spheres with $r_{1}^{2}+r_{2}^{2}=1$.

Conversely, if $M$ is a small sphere $S^{n-1}(r) \subset S^{n}$ for some $r \in(0,1]$, then together with (26), Lemma 1 shows that $M$ satisfies the geodesic chord property. If $M$ is the product $S^{p}\left(r_{1}\right) \times S^{n-p-1}\left(r_{2}\right) \subset$ $\mathbb{E}^{p+1} \times \mathbb{E}^{n-p}$ with $r_{1}^{2}+r_{2}^{2}=1$, then at $x=\left(x_{1}, x_{2}\right) \in \mathbb{E}^{p+1} \times \mathbb{E}^{n-p}$ we have

$$
G\left(x_{1}, x_{2}\right)=\frac{1}{\sqrt{r_{1}^{2}+r_{2}^{2}}}\left(\frac{r_{2}}{r_{1}} x_{1},-\frac{r_{1}}{r_{2}} x_{2}\right) .
$$

Hence, it follows from Lemma 1 that $M$ satisfies the geodesic chord property.

Summarizing the above discussions, we get the classification theorem as follows.

Theorem 1. For a hypersurface $M$ in the unit hypersphere $S^{n} \subset \mathbb{E}^{n+1}$, the following are equivalent:

1. M satisfies the geodesic chord property.

2. The Gauss map $G$ satisfies $|\langle G(x), y\rangle|=|\langle G(y), x\rangle|$ for arbitrary $x, y \in M$.

3. The Gauss map $G$ satisfies $G(x)=A x+b$ for an $(n+1) \times(n+1)$ matrix $A$ and a vector $b \in \mathbb{E}^{n+1}$.

4. $M$ is an open portion of either a sphere $S^{n-1}(r)$ or a product $S^{p}\left(r_{1}\right) \times S^{n-p-1}\left(r_{2}\right)$ of spheres with $r_{1}^{2}+r_{2}^{2}=1$.

\section{Hypersurfaces in the Hyperbolic Space}

In this section, we consider a hypersurface $M$ in the hyperbolic space $H^{n}$ which lies in the $(n+1)$-dimensional Minkowski space $\mathbb{E}_{1}^{n+1}$

First of all, let us recall some preliminaries. We consider the $(n+1)$-dimensional Minkowski space $\mathbb{E}_{1}^{n+1}$ with metric $d s^{2}=d x_{1}^{2}+\cdots+d x_{n}^{2}-d x_{n+1}^{2}$, where $x=\left(x_{1}, \cdots, x_{n+1}\right)$. In other words, for $x, y \in$ $\mathbb{E}_{1}^{n+1}$ we use the Lorentzian scalar product $\langle x, y\rangle_{1}=x_{1} y_{1}+\cdots+x_{n} y_{n}-x_{n+1} y_{n+1}$. Let us denote by $H^{n}(r) \subset \mathbb{E}_{1}^{n+1}$ the spacelike hyperquadric defined by $\langle x, x\rangle_{1}=-r^{2}$ with $x_{n+1}>0$. Then $H^{n}(r)$ is a Riemannian space form with constant sectional curvature $K=-\frac{1}{r^{2}}$. When $r=1, H^{n}(1)$ is called the standard imbedding of the hyperbolic space $H^{n}$ of curvature $K=-1$, or simply the hyperbolic space (cf. $[13,14])$.

We introduce a notation for the Lorentzian scalar product. For a vector $v=\left(v_{1}, \ldots, v_{n+1}\right) \in \mathbb{E}_{1}^{n+1}$, we put $\bar{v}=\left(v_{1}, \ldots, v_{n},-v_{n+1}\right)$. Then for any $v, w \in \mathbb{E}_{1}^{n+1}$, we have $\langle v, w\rangle_{1}=\langle v, \bar{w}\rangle$, where $\langle\cdot, \cdot\rangle$ denotes the Euclidean scalar product. For an $(n+1) \times k$ matrix $A=\left[A_{1}, \ldots, A_{k}\right]$ with column vectors $A_{1}, \ldots, A_{k}$, we let $\bar{A}=\left[\bar{A}_{1}, \ldots, \bar{A}_{k}\right]$. Then we have $(\bar{A} v)=\bar{A} v$ for any $k$-dimensional vector $v$.

For a point $x \in M$, we denote by $G(x)$ the unit normal to $T_{x} M$ in the hyperbolic space $H^{n}$. Then $G$ is called the Gauss map of $M$ in the hyperbolic space $H^{n}$. For an orthonormal local frame field $\left\{e_{1}(x), \ldots, e_{n-1}(x)\right\}$ on $M,\left\{e_{1}(x), \ldots, e_{n-1}(x), G(x), x\right\}$ forms an orthonormal basis of $T_{x} \mathbb{E}_{1}^{n+1}$ with respect to the Lorentzian scalar product. We denote by $A(x)$ the $(n+1) \times(n-1)$ frame matrix $\left[e_{1}(x), \ldots, e_{n-1}(x)\right]$ with column vectors $e_{1}(x), \ldots, e_{n-1}(x)$. Then, we have for the frame matrix $A(x)$

$$
A(x)^{t} \bar{A}(x)=I_{n-1}
$$

and

$$
A(x) \bar{x}=\bar{A}(x) x=0 .
$$

For any two points $x, y \in M \subset H^{n}$, we have $\langle x, y\rangle_{1}=\langle x, \bar{y}\rangle\left\langle-1\right.$. Hence $\langle x, y\rangle_{1}=-\cosh \theta$ for some positive $\theta$, which is called the hyperbolic angle between $x$ and $y$ ([14], p. 144). Then the unit speed geodesic chord $\gamma(t)$ with $\gamma(0)=x$ and $\gamma(\theta)=y$ is given by $\gamma(t)=\cosh t x+\sinh t v$, where

$$
v=v_{x, y}=-\operatorname{coth} \theta x+\operatorname{csch} \theta y
$$


Note that the geodesic chord $\gamma(t)$ has initial velocity $v=v_{x, y}$ at $x$. The angle between $v$ and the tangent space $T_{x} M \subset T_{x} H^{n}$ is defined by the angle between $v$ and the projection $P_{x}(v)$ of $v$ into $T_{x} M$. Hence, if we let $\phi$ denote the angle between the geodesic chord $\gamma$ from $x$ to $y$ and the tangent plane $T_{x} M$ at the point $x$, then we have

$$
\cos \phi=\left|P_{x}(v)\right|=\sqrt{\left\langle P_{x}(v), P_{x}(v)\right\rangle_{1}} .
$$

By a similar argument to that in Section 2, using (29)-(32) we may prove the following two lemmas. We omit the proofs.

Lemma 3. For a hypersurface $M$ in the hyperbolic space $H^{n}$, the following are equivalent:

1. M satisfies the geodesic chord property.

2. For any two points $x, y \in M$, the frame matrix $A$ of $M$ satisfies

$$
\left\langle A(x)^{t} \bar{y}, A(x)^{t} \bar{y}\right\rangle=\left\langle A(y)^{t} \bar{x}, A(y)^{t} \bar{x}\right\rangle,
$$

where $\langle\cdot, \cdot\rangle$ is the Euclidean inner product.

3. For any two points $x, y \in M$, the Gauss map $G(x)$ of $M$ satisfies

$$
\langle G(x), y\rangle_{1}=\epsilon\langle x, G(y)\rangle_{1}
$$

where $\epsilon= \pm 1$.

Lemma 4. Suppose that a hypersurface $M$ in the hyperbolic space $H^{n} \subset \mathbb{E}_{1}^{n+1}$ satisfies the geodesic chord property. If the hypersurface $M$ is full in $\mathbb{E}_{1}^{n+1}$, then for an $(n+1) \times(n+1)$ matrix $A$, the Gauss map $G(x)$ satisfies $G(x)=A x$.

If a hypersurface $M \subset H^{n}$ is not full in $\mathbb{E}_{1}^{n+1}$, then $M$ is contained in a hyperplane $P=\{x \in$ $\left.\mathbb{E}_{1}^{n+1} \mid\langle x, a\rangle=c\right\}$ for some nonzero $a \in \mathbb{E}_{1}^{n+1}$ and some constant $c$.

We divide by three cases according to the causal character of the nonzero vector $a \in \mathbb{E}_{1}^{n+1}$.

Case 1. Suppose that $\langle a, a\rangle<0$. Then, up to congruences of $H^{n}$ we may assume that $a=(0, \ldots, 0,1)$. Hence we have for some $\theta$

$$
M \subset\left\{x \in H^{n} \mid x_{n+1}=\cosh \theta\right\} .
$$

Thus, $M$ is an open part of the hypersphere $S^{n-1}(\sinh \theta)$ in the Euclidean space $\mathbb{E}^{n} \subset \mathbb{E}_{1}^{n+1}$. In this case, we have

$$
G(x)=-\operatorname{coth} \theta x+\operatorname{csch} \theta a .
$$

This shows that $M$ satisfies $G(x)=A x+b$ for $A=-\operatorname{coth} \theta I$ and $b=\operatorname{csch} \theta a$.

Case 2. Suppose that $\langle a, a\rangle>0$. Then, up to congruences of $H^{n}$ we may assume that $a=(1,0, \ldots, 0)$. Hence we have for some $\theta$

$$
M \subset\left\{x \in H^{n} \mid x_{1}=\sinh \theta\right\} .
$$

Thus, $M$ is an open part of the hyperbolic space $H^{n-1}(\cosh \theta)$ in the Minkowski space $\mathbb{E}_{1}^{n} \subset \mathbb{E}_{1}^{n+1}$. In this case, we have

$$
G(x)=\tanh \theta x+\operatorname{sech} \theta a,
$$

which shows that $M$ satisfies $G(x)=A x+b$ for $A=\tanh \theta I$ and $b=\operatorname{sech} \theta a$.

Case 3. Suppose that $\langle a, a\rangle=0$. Then, up to congruences of $H^{n}$ we may assume that $a=(0, \ldots, 0,1,1)$ and $c=-1$. Hence we have

$$
M \subset N=\left\{x \in H^{n} \mid x_{n+1}=x_{n}+1\right\} .
$$


Note that $N=\left\{(x, f(x), f(x)+1) \mid x \in \mathbb{E}^{n-1}\right\} \subset H^{n}$, where $f(x)=\frac{1}{2}|x|^{2}$ for $x=\left(x_{1}, \ldots, x_{n-1}\right) \in$ $\mathbb{E}^{n-1}$. In this case, we have

$$
G(x)=x-a,
$$

which shows that $M$ satisfies $G(x)=A x+b$ for $A=I$ and $b=-a$.

Finally, we use the following proposition ([12]).

Proposition 3. A hypersurface $M$ in $H^{n} \subset \mathbb{E}_{1}^{n+1}$ satisfies $G(x)=A x+b$ if and only if $M$ is isoparametric, or equivalently $M$ is an open piece of one of the following hypersurfaces:

1. $S^{n-1}(\sinh \theta) \subset H^{n}$,

2. $H^{n-1}(\cosh \theta) \subset H^{n}$,

3. $S^{p}(\sinh \theta) \times H^{n-p-1}(\cosh \theta) \subset H^{n}$,

4. $N=\left\{\left.(x, f(x), f(x)+1)\left|f(x)=\frac{1}{2}\right| x\right|^{2}, x \in \mathbb{E}^{n-1}\right\} \subset H^{n}$.

Summarizing the above discussions, we prove the following classification theorem.

Theorem 2. A hypersurface $M$ in $H^{n} \subset \mathbb{E}_{1}^{n+1}$ satisfies the geodesic chord property if and only if it is an open piece of one of the hypersurfaces in Proposition 3.

Proof. Together with Lemma 3, it follows from (36), (38) and (39) that the hypersurfaces $S^{n-1}(\sinh \theta)$, $H^{n-1}(\cosh \theta)$ and $N$ satisfies the geodesic chord property, respectively. Hence, it remains to show that $S^{p}(\sinh \theta) \times H^{n-p-1}(\cosh \theta) \subset H^{n}$ satisfies the geodesic chord property.

Suppose that $M=S^{p}(\sinh \theta) \times H^{n-p-1}(\cosh \theta)$. Then, for a point $x=\left(x_{1}, x_{2}\right) \in S^{p}(\sinh \theta) \times$ $H^{n-p-1}(\cosh \theta)$ we have

$$
G(x)=\left(\operatorname{coth} \theta x_{1}, \tanh \theta x_{2}\right) .
$$

Thus, Lemma 3 shows that $M$ satisfies the geodesic chord property. This completes the proof.

From the proof of Theorem 2, we also obtain

Theorem 3. For a hypersurface $M$ in the hyperbolic space $H^{n} \subset \mathbb{E}_{1}^{n+1}$, the following are equivalent:

1. M satisfies the geodesic chord property.

2. The Gauss map $G$ satisfies $\left|\langle G(x), y\rangle_{1}\right|=\left|\langle G(y), x\rangle_{1}\right|$ for any $x, y \in M$.

3. The Gauss map $G$ satisfies $G(x)=A x+b$ for an $(n+1) \times(n+1)$ matrix $A$ and a vector $b \in \mathbb{E}_{1}^{n+1}$.

4. $M$ is an isoparametric hypersurface of $H^{n}$.

5. $M$ is an open part of one of the following hypersurfaces: $S^{n-1}(r), H^{n-1}(r), S^{p}\left(r_{1}\right) \times H^{n-p-1}\left(r_{2}\right), N$, where $r_{2}^{2}-r_{1}^{2}=1$ and $N=\left\{\left(x, \frac{1}{2}|x|^{2}, \frac{1}{2}|x|^{2}+1\right) \mid x \in \mathbb{E}^{n-1}\right\}$.

\section{Conclusions}

In this paper, we have classified hypersurfaces in the nonflat space forms satisfying the so-called geodesic chord property. As a result, we have shown that such a spherical hypersurface is an open portion of either a sphere or a product of two spheres, which are isoparametric spherical ones with (at most) two principal curvatures. For hypersurfaces in the hyperbolic space, we have proven that the geodesic chord property is another characterization of isoparametric ones. We hope the results will help studying hypersurfaces in the nonflat space forms.

Author Contributions: D.-S.K. and Y.H.K. set up the problem and computed the details and D.W.Y. checked and polished the draft. These authors contributed equally to this work.

Funding: This research was supported by Basic Science Research Program through the National Research Foundation of Korea (NRF) funded by the Ministry of Education (NRF-2018R1D1A3B05050223). 
Acknowledgments: We are very thankful to the reviewers for their suggestions to improve the quality of this paper.

Conflicts of Interest: The authors declare no conflict of interest.

\section{References}

1. Rademacher, H.; Toeplitz, O. The Enjoyment of Mathematics; Translated from the Second (1933) German Edition and with Additional Chapters by H. Zuckerman; Princeton Science Library, Princeton University Press: Princeton, NJ, USA, 1994.

2. Chen, B.-Y.; Kim, D.-S.; Kim, Y.H. New characterization of W-curves. Publ. Math. Debr. 2006, 69, 457-472.

3. Kim, D.-S.; Kim, Y.H. New characterizations of spheres, cylinders and W-curves. Linear Algebra Appl. 2010, 432, 3002-3006. [CrossRef]

4. Boas, H.P. A geometric characterization of the ball and the Bochner-Martinelli kernel. Math. Ann. 1980, 248, 275-278. [CrossRef]

5. Boas, H.P. Spheres and cylinders: A local geometric characterization. Ill. J. Math. 1984, 28, 120-124. [CrossRef]

6. Wegner, B. A differential geometric proof of the local geometric characterization of spheres and cylinders by Boas. Math. Balk. (N.S.) 1988, 2, 294-295.

7. Kim, D.-S. Ellipsoids and Elliptic hyperboloids in the Euclidean space $E^{n+1}$. Linear Algebra Appl. 2015, 471, 28-45. [CrossRef]

8. Kim, D.-S.; Kim, Y.H. Some characterizations of spheres and elliptic paraboloids. Linear Algebra Appl. 2012, 437, 113-120. [CrossRef]

9. Kim, D.-S.; Kim, Y.H. Some characterizations of spheres and elliptic paraboloids II. Linear Algebra Appl. 2013, 438, 1356-1364. [CrossRef]

10. Kim, D.-S.; Song, B. A characterization of elliptic hyperboloids. Honam Math. J. 2013, 35, 37-49. [CrossRef]

11. Kim, D.-S.; Kim, Y.H.; Yoon, D.W. On standard imbeddings of hyperbolic spaces in the Minkowski space. Comptes Rendus Math. 2014, 352, 1033-1038. [CrossRef]

12. Kim, D.-S. On the Gauss map of Hypersurfaces in the space form. J. Korean Math. Soc. 1995, 32, 509-518.

13. Kühnel, W. Differential Geometry, Curves-Surfaces-Manifolds; Translated from the 1999 German Original by Bruce Hunt; Student Mathematical Library, 16; American Mathematical Society: Providence, RI, USA, 2002.

14. O'Neill, B. Semi-Riemannian Geometry with Applications to Relativity; Pure and Applied Mathematics, 103; Academic Press, Inc.: New York, NY, USA, 1983. 